\title{
Costruire linee del tempo nella scuola primaria
}

\author{
di Gianluca Gabrielli
}

Nov 11, 2019 | In evidenza, Pensare la didattica $|\underline{0}|$

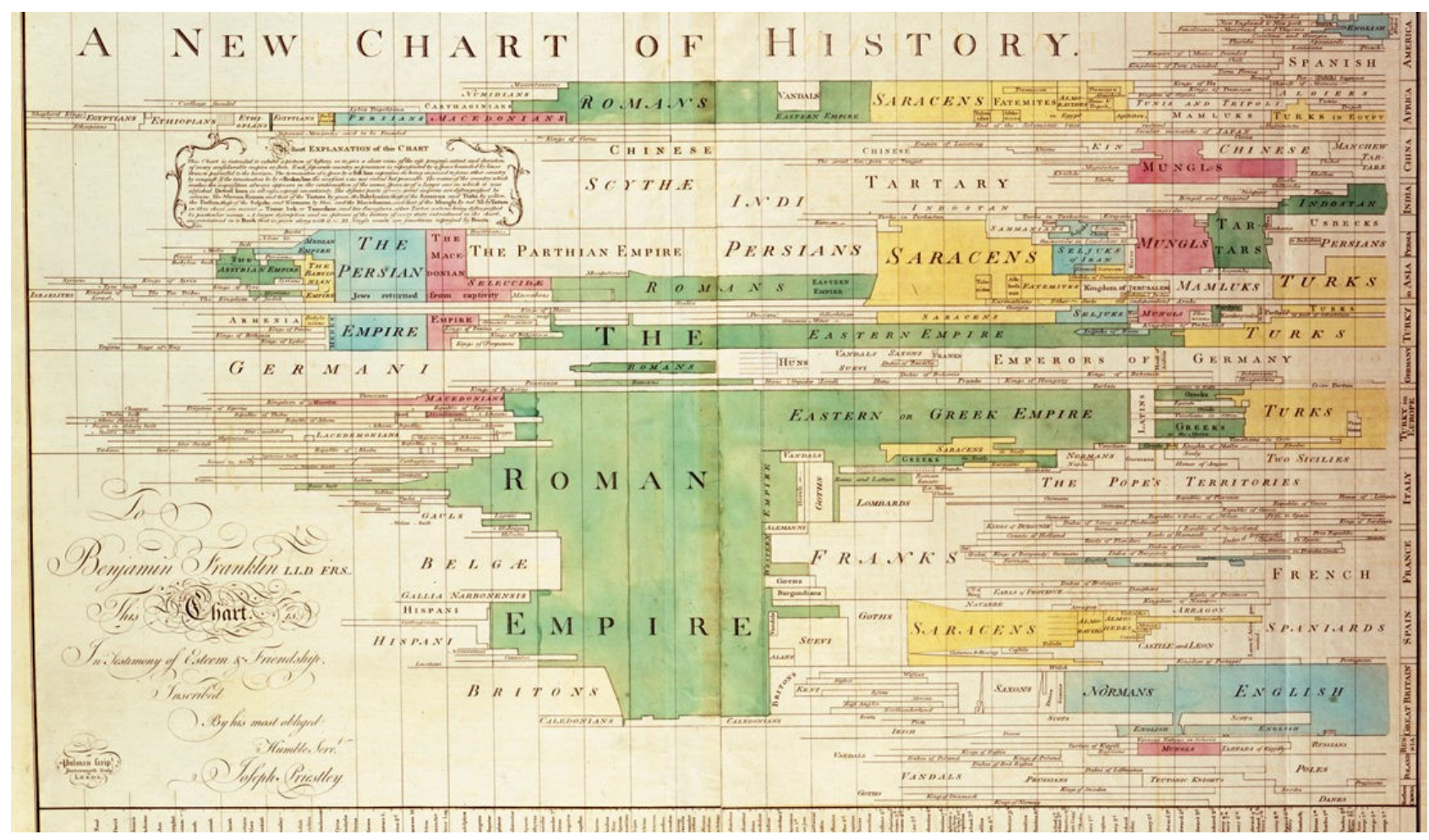

Di Alan Jacobs - http://www.thenewatlantis.com/publications/history-as-wall-art, Pubblico dominio, Collegamento

\begin{abstract}
L'uso e soprattutto la costruzione di linee del tempo nella scuola primaria costituiscono un campo di lavoro potenzialmente ricco, ma spesso trascurato dagli insegnanti. Partendo dalle suggestioni della ricerca di Rosenberg e Grafton sulla storia delle Cartografie del tempo, l'intervento intende riflettere sulle forme che assume la rappresentazione del passato e del tempo nella scuola per arrivare a presentare alcune ipotesi di lavoro da proporre nelle classi.

La costruzione dell'immagine del passato, la sua mappatura mentale, la stessa concezione della storia passa anche attraverso le forme concrete di rappresentazione cronologica che si sono sviluppate e avvicendate nel corso del tempo. La storia della mappatura storica (almeno di quella occidentale) è stata studiata nel bel volume di Rosenberg e Grafton Cartografie del tempo. Ricco di ben trecento splendide immagini patinate, il libro presenta un'incredibile carrellata di tabelle, diagrammi, rappresentazioni iconiche e simboliche del passato per come è stato rappresentato negli ultimi duemila anni. Una storia per nulla lineare, nella quale gli autori hanno individuato formalizzazioni che incarnano delle vere e proprie svolte periodizzanti. Tale è sicuramente, nel IV secolo dopo Cristo, la protocristiana Cronaca di Eusebio, una tabella con gli anni disposti sull'asse verticale, prodotta e divenuta punto di riferimento per svariati secoli. La grande svolta successiva ci
\end{abstract}


viene indicata nell'innovazione di Jeffrey Priestley che nel secolo dei lumi produce la Biography chart, un diagramma in cui ogni biografia di un personaggio importante viene rappresentata come un segmento collocato su una striscia orizzontale suddivisa omogeneamente in secoli, dando la possibilità all'osservatore di avere sott'occhio in modo intuitivo le contemporaneità e le relazioni temporali tra i diversi personaggi. Tra questi due punti di riferimento, molteplici formalizzazioni prendono corpo nel tentativo di trasformare il passato in qualcosa di visibile e non solo leggibile, di fornire supporti ai fini della memorizzazione, di convogliare informazioni da diverse tradizioni storiografiche per renderle coerenti e fruibili in maniera evidente e visiva.

Si tratta di una vicenda troppo ricca e affascinante per non cedere alla tentazione di ricordare qui alcuni altri esempi. Ad esempio Johann Buno, teologo e pedagogo tedesco del Seicento, scelse di dare spazio alla rappresentazione allegorica del passato per sostenere lo sforzo della memorizzazione degli studenti, attribuendo a ognuno dei quattro millenni - dalla creazione all'avvento di Cristo - una immagine esemplare: un'aquila, un fascio di assi a ricordare l'arca di Noè, un cammello e un drago, e su questi sfondi memorabili distribuì i disegni dei personaggi impegnati in azioni anch' esse allegoriche (Tolomeo - da astronomo - venne, ad esempio, rappresentato con lo sguardo rivolto al cielo). Nell'Ottocento, in epoca romantica, la storia potè prendere la forma di una corrente che scorreva lungo il tempo divaricandosi e riunendosi, come un insieme di torrenti colorati che si incrociavano a partire dalla tempesta sulla sommità del manifesto di William Bell. O come l'albero di Stephen e Daniel Dod che da un'unica radice cresceva ramificandosi, poi riunendosi e quindi di nuovo dando origine a una ventina di rami. Infine con la seconda metà dell'Ottocento, in epoca positivista (e imperialista), il tempo si fa omogeneo, matematico, disciplinatore e apparentemente oggettivo e che si mostra attraverso un profluvio di grafici e diagrammi capaci di rappresentare il passato in una forma talmente meccanica che rischia sempre di apparire aproblematica, indiscutibile, falsamente «esatta».

\section{L'immagine del tempo in cui siamo immersi}

Noi insegnanti di storia del XXI secolo siamo eredi di questa tradizione e soprattutto ci muoviamo in un immaginario fortemente permeato dalle formule visive che, a partire da Priesltey, si sono affermate negli ambiti più vari e che a volte appaiono quasi naturali, come se non fossero costate centinaia di anni di storia e di conflitti culturali. Senza contare che sembrano la sola formalizzazione «corretta», mentre non costituiscono certo un risultato «obbligato» o «scientifico» della riflessione sul passato, ma la forma che a oggi è risultata vittoriosa a valle di un serrato e plurisecolare dibattito intellettuale.

Così nel nostro tempo noi adulti osserviamo i diagrammi che mostrano l'andamento mensile o decennale dello spread, un elettrocardiogramma, l'accelerazione recente del riscaldamento globale o l'incremento della forbice tra i morti civili e militari nelle guerre degli ultimi secoli con una naturalezza dovuta all'immersione profonda in queste forme di rappresentazione della storia che hanno conquistato le nostre menti e il nostro modo di "vedere" il passato. Da adulti dobbiamo certo diffidare dell'immagine apodittica che ci forniscono, non solo perché ogni grafico può contenere retoriche funzionali a questa o a quella trasmissione di informazioni ammantate di oggettività, ma essenzialmente perché il passato che ci presentano non è indiscutibile né scontato, e non costituisce una predizione all'indietro nel tempo prodottasi senza alternative, né una premessa vincolante del presente. D'altra parte, una volta ricordate queste avvertenze essenziali, possiamo ugualmente servirci della familiarità con queste forme di rappresentazione per organizzare il nostro sguardo sul passato, per articolare su queste strisce di storia ogni nuova informazione e ragionamento che abbia una dimensione diacronica. 
Nei bambini invece questo sfondo integratore delle acquisizioni di ciò che chiamiamo «passato» è tutto da costruire, sia attraverso l'assorbimento degli elementi inconsapevoli e involontari che giungono dall'universo della comunicazione, sia con azioni consapevoli da organizzare in classe. Nelle prime classi è abbastanza comune sentire domande come «Maestro, tu c'eri quando c'erano i dinosauri?», o «L'hai fatta la guerra mondiale?». La profondità della prospettiva temporale è il risultato auspicabile di un percorso da dispiegare nel tempo, pazientemente, senza fretta. È vero, però, che fin da quell'età i bambini sono carichi di "immagini del passato" che attraverso la televisione, il cinema e la vita di relazione entrano nella loro mente e prendono una forma difficile da decodificare per gli adulti, ma che è sicuramente carica di riferimenti ad un'idea del passato in formazione.

Maestre e maestri possono fornire un aiuto a queste piccole menti cariche di elementi di storia affinché diano loro un ordine, per trovare loro uno sfondo, una narrazione. Quando queste immagini del passato trovano un'arena di ascolto interessata e curiosa sono capaci di produrre discussioni che non si sa dove possano portare, ma che sono affascinanti finestre su quelle giovani menti. Una delle forme che possono prendere queste riflessioni fatte in classe è la linea o striscia del tempo. 


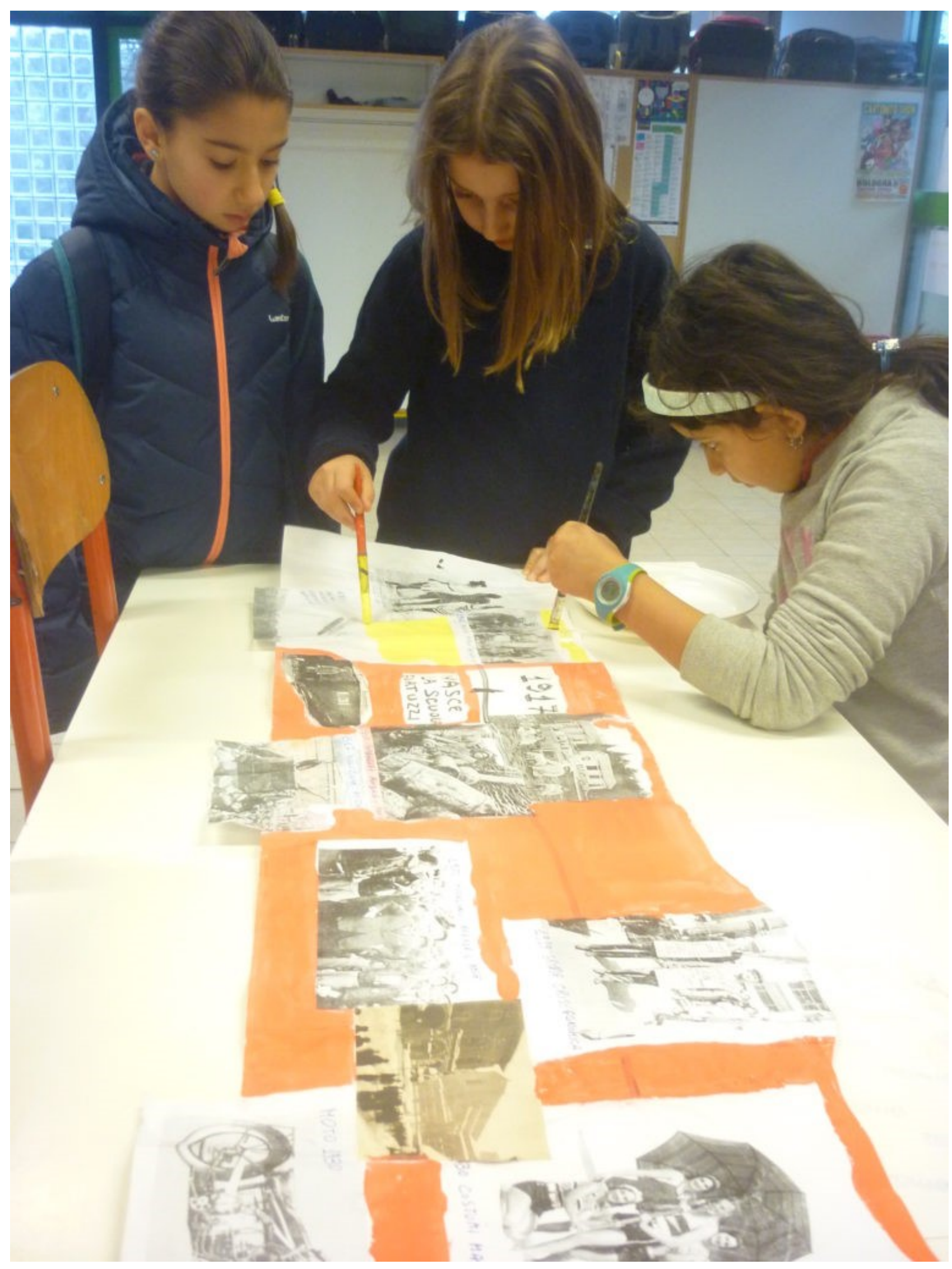

Le linee del tempo non sono certo un oggetto sconosciuto nella didattica della scuola primaria. Nei kit allegati a ogni adozione di libri di testo compare immancabilmente una versione murale a stampa della linea da appendere in classe e che rappresenta le "civiltà" prese in considerazione nel curricolo di storia. Tuttavia, a prescindere dalla loro qualità grafica e di contenuti, queste linee del tempo 
poche volte vengono appese, e anche quando campeggiano sulle pareti raramente vengono "viste" e divengono un "ausilio didattico". Il loro grande difetto, da cui non possono sfuggire, è che sono preparate fuori dalla classe.

Una linea del tempo condensa concetti complessi in rappresentazioni che li sintetizzano, quindi vanno comprese. Se la preparazione è fatta senza la partecipazione di bambini e bambine il risultato sarà sostanzialmente inutile, finiranno per essere ignorate $\mathrm{o}$, al più, apprese senza essere comprese. Insomma le linee del tempo fornite dagli editori sono un oggetto scolastico passivo che cerca di comprendere per "esposizione" un processo di sintesi fatto da altri. Per rendere nuovamente efficace la striscia del tempo bisogna che la pratica didattica trasferisca l'azione intellettuale di progettare e costruire mappe di storia dagli autori di sussidiari alle bambine e ai bambini, mettendoli al lavoro, mani e intelletto.

\section{Le prime classi: il tempo della giornata a scuola}

La frequenza a scuola costituisce una formidabile palestra di percezione della linearità e ciclicità delle attività giornaliere. L'organizzazione della vita scolastica, infatti, si ripete con regolarità per consentire agli adulti - insegnanti e collaboratori scolastici - di utilizzare al meglio i tempi e gli spazi della scuola ma anche per fornire ai bambini e alle bambine una cornice spazio-temporale stabile in cui entrare e uscire ogni giorno senza perdersi. Così le prime formalizzazioni del tempo possono nascere per rappresentare la giornata, il planning scolastico. Solitamente conviene iniziare fissando l'entrata e l'uscita - terminus a quo e ad quem della giornata scolastica - su una striscia di carta che viene subito appesa, meglio se a portata di mani e di sguardo di bambino; poi la discussione procede per individuare le differenti fasi di attività e ritualità, la loro rappresentazione attraverso disegni o fotografie, quindi la dislocazione concreta di queste immagini seguendo una cronologia relativa («prima questa, poi quella») senza badare alle scansioni orarie e senza intervenire sugli errori (che - se presenti - vanno individuati dai bambini stessi nel corso del tempo).

Dopo questa prima fase di costruzione la linea va introiettata; per facilitare tale processo occorre che chi insegna vi faccia riferimento più spesso possibile nel corso della vita scolastica («Abbiamo finito la lezione, quindi adesso andremo a mangiare», indicando la linea) e che si colgano tutte le occasioni per arricchire e precisare la successione («Prima di mangiare ci si lavano le mani, quindi aggiungiamo questa azione nella successione al punto giusto; prima di uscire indossiamo le giacche: chi fa il disegno per aggiungere anche questa azione?»). Per evitare che questa linea del tempo si trasformi da descrittiva a normativa vanno valorizzati tutti gli interventi che introducono eccezioni («Oggi non abbiamo giacche, così saltiamo dalla merenda all'uscita perché è caldo»).

Ulteriori stimoli in questa direzione possono emergere andando in seguito ad esplorare - anche nelle classi successive - ciò che accade prima e dopo la permanenza a scuola (ad esempio il mattino, dal risveglio all'entrata in classe). In questo modo la linea diviene plurale, include diversi "bambini-continenti" che vivono azioni in parte uguali e in parte diverse, alcune organizzate allo stesso modo e altre diversamente (colazione in pigiama e poi bagno oppure bagno, vestirsi e poi colazione? Televisione sì o no e prima o dopo della colazione? Durante?). Una pluralità di varianti che confluiscono tutte nell'entrata in classe e che diviene piacevole e utile rappresentare in parallelo, come nella Biography chart di Priestly, collocandole in continuità con la striscia della giornata scolastica.

\section{Le prime classi: la vita dei viventi}

Ogni essere vivente nasce e muore. Prendiamo un fagiolo e un delfino. Apriamo anche su di loro la discussione: ci sono dei momenti particolari nella loro vita che possiamo individuare? Solitamente 
le "fasi" della vita, questi "elementi periodizzanti", emergono dalle riflessioni dei bimbi e vanno colte, trasformate in frase e disegno e poste in successione in una striscia del tempo di vita del fagiolo e in un'altra striscia dedicata al delfino. Solitamente emerge dalla discussione la nascita dei figli dei soggetti al centro del ragionamento; ciò che crea le condizioni per aggiungere altre strisce del tempo alle precedenti. Il succedersi delle generazioni, i nonni-fagiolo e i bisnonni-delfino hanno diritto alla loro striscia e il problema allora diviene come collegare le une alle altre, come rappresentare in maniera approssimativa queste catene di generazioni («Attacchiamo altre strisce alla prima? E poi altre ancora? Dividiamoci le strisce da disegnare, un gruppo disegna la vita del fagiolo mamma, le dà un nome e ne inventa la storia; un altro disegna quella di un figlio, un altro ancora quella di una figlia del figlio...»). Infine l'atto di collegarle: nel segmento della vita del delfino a un certo punto si diparte la striscia del figlio, produce una specie di grande diagramma ad albero, un torrente come quelli che rappresentavano la visualizzazione della storia nell'Ottocento, una saga famigliare riferita al fagiolo che negli anni seguenti può essere ritoccata attribuendogli una cronologia assoluta («Quanti anni vive il fagiolo? Pianta annuale, ogni generazione un anno. Quanti anni vive il delfino? Quanto dura la sua gestazione? A che età può riprodursi? Se il nostro delfino è nato nel 2000, che anno segniamo in corrispondenza del suo primo nato? E invece per il fagiolo?»).

\section{La storia generale}

Una serie di vicende che si sono prodotte negli ultimi vent'anni ha fatto sì che nella scuola primaria, in quarta e quinta classe, si insegni la storia antica. È vero che le Indicazioni nazionali non sono prescrittive dei contenuti, ma è innegabile che dal combinato che scaturisce dalle frasi incluse nelle Indicazioni del 2012 e dall'operato degli autori dei libri di testo emerge in maniera chiara questo curricolo di storia generale. Ciò produce una serie di problemi. Da una parte i ragazzi rischiano di concludere questo percorso di cinque anni senza avere una visione complessiva - anche se generica - della storia dell'umanità. Dall'altra la contemporaneità stessa esce dall'attenzione degli insegnanti della scuola primaria, facendo sì che la storia degli ultimi 120 anni sia proposta allo sguardo dei bambini e delle bambine solo come celebrazione di date (la Shoah, il 25 aprile, ecc...), con il forte rischio di non riuscire a fornire un'abbozzata descrizione generale dell'ultimo secolo in cui collocare - almeno provvisoriamente - le emergenze di passato e della storia che giungono dal mondo dei media e dalle discussioni in classe, in particolare nelle classi terminali.

Il rischio è di arrivare fino a 11 anni senza acquisire una immagine generale del passato - magari approssimativa ma comunque attiva - cui fare riferimento quando si incontrano le tracce e le sopravvivenze della storia. $\mathrm{O}$, per dire meglio, si rischia di arrivare a 11 anni con immagini del passato costruite al di fuori della scuola, assemblando e connettendo aspetti del passato senza avere un luogo di confronto, discussione, costruzione concreta di questa immagine insieme ai compagni e ai docenti. Come provare a fronteggiare questa difficoltà presente nei curricoli attuali della primaria?

\section{Una linea del tempo storico}

Anche in questo caso può essere d'aiuto la striscia del tempo, da intendersi non come panacea ma come catalizzatore di discussione, riflessione, confronto e progressiva costruzione di uno sfondo integratore di tipo storico.

Non importa attendere le classi alte per sondare quali immagini del passato abitano i bambini e le bambine. È utile pensare attività di discussione per farle emergere anche subito, in prima o seconda classe. «Cosa conosci del passato? Cosa sai della storia? Cos'era un tempo diverso da oggi? Conosci animali o personaggi che oggi non ci sono più?». 
È utile partire dalla foto di classe per individuare il «presente» e quindi includere nel «passato» tutto ciò che è accaduto «prima». Queste discussioni sono occasioni importanti sia per l'insegnante che può prendere le misure del «sapere storico» circolante tra $\mathrm{i}$ banchi, sia per $\mathrm{i}$ bambini e le bambine che sono indotti ad organizzare le loro conoscenze sul passato, a discriminarle, a sceglierle e a metterle in comune. Anche qui la trasformazione in disegno è il mezzo più semplice, immediato e fondamentale per trasformare un pensiero in un oggetto, reificarlo in senso buono. Il gioco poi può continuare anche a casa, allargandolo ai compiti e quindi ai genitori, che andrebbero, però, avvertiti come non si tratti di «insegnare la storia» e gli avvenimenti del passato ai propri figli, ma di estendere il brainstorming di classe alla cena casalinga, facendo mente locale su altri elementi del passato che dovessero emergere nella vita a casa, nella televisione, nel fine settimana.

La discussione può anche finire in un cartellone in cui piazzare provvisoriamente i nostri «elementi di passato», e tuttavia sarebbe meglio fin da subito dare una strutturazione lineare a questo passato magmatico. Qui, a differenza della linea della giornata scolastica, abbiamo un punto di riferimento che è la foto di classe, il presente, e una striscia del tempo che si dispiega a sinistra, all'indietro, nel passato, che è pronta ad accogliere i nostri elementi. Ma dove piazzarli? Come piazzarli gli uni rispetto agli altri? È questo il momento per aprire una nuova discussione tra bambini e bambine, ancora una volta ponendosi come insegnanti nella posizione di chi registra le diverse opinioni, le accetta tutte, e se capita sostiene quelle che si mostrano potenzialmente utili a muovere il gruppo verso una razionalizzazione della cronologia relativa. Non è quindi un problema se nella prima versione della linea del tempo gli elementi del passato non hanno la giusta collocazione: si può tranquillamente attendere l'occasione per tornare su un elemento e riaprire la discussione, con nuovi elementi che aiutino a correggere la prima ipotesi. In questo modo insieme all'acquisizione di alcuni elementi di datazione relativa della storia generale - ad esempio che le guerre con gli aerei tendenzialmente sono da porre dopo quelle con la spada - $\mathrm{i}$ bambini dovrebbero intuire che la stessa striscia del tempo è una costruzione degli storici che è soggetta a continue rettifiche e modifiche man mano che crescono gli elementi di conoscenza del passato.

\section{Le classi alte: le date e le periodizzazioni}

Se si parte nella costruzione della striscia nelle classi più alte credo che le modalità di azione non debbano cambiare sostanzialmente. L'unica differenza notevole riguarda l'intervento dei docenti che può essere più incisivo, arricchendo gli elementi segnalati dai bambini con altri elementi storici introdotti come importanti elementi di conoscenza. Lavorando in una quarta, ad esempio, non è pensabile che si eviti di parlare della scoperta (conquista) dell'America solo perché non esce dal brainstorming dei bambini: va introdotta e spiegata nella sua importanza. A questo punto, infatti, gli elementi del passato possono venire inquadrati su due livelli, poiché alcuni episodi possono essere segnalati come eventi periodizzanti.

L'evento periodizzante ci aiuta a suddividere il passato in periodi, interpretandolo. Prima della scoperta-conquista dell'America due popoli vivevano senza contatti tra loro, dopo l'evento periodizzante non furono più indipendenti. Questa introduzione della periodizzazione è fondamentale, perché se gli avvenimenti storici sono praticamente infiniti, gli avvenimenti periodizzanti sono da scegliere attraverso un'interpretazione delle conseguenze che si collegano solo ad alcuni di questi avvenimenti. Nel Novecento le due guerre mondiali sono state fortemente periodizzanti, mentre la forza periodizzante del naufragio del Titanic è certamente minore: anche se quest'ultimo avvenimento va per la maggiore tra i banchi di scuola, è importante che comprendano che esso sta dentro una fase storica e non la forgia.

Nelle linee del tempo messe a punto nelle classi alte è giusto che compaiano le date, ora rese comprensibili anche dal parallelo sviluppo del curricolo di matematica. Non occorre però 
riorganizzare matematicamente la linea, cercando la proporzionalità delle distanze: è più importante dare maggiore risalto agli avvenimenti che si decide di riconoscere come periodizzanti piuttosto che affaticarsi in una schematizzazione astratta del passato secondo l'unità di misura temporale del secolo.

Se non sarà emersa già nei primi anni sulla base delle diverse genealogie culturali che sono presenti nelle classi, sicuramente in quarta si affronterà la questione della relativizzazione della storia del cosiddetto Occidente.

Nel curricolo ministeriale, infatti, lo studio delle civiltà antiche comprende le società dell'Indo e del fiume Giallo, affrontate parallelamente e contemporanee a quelle del bacino mediterraneo. Questa è l'occasione per esplorare con la classe un altro limite intrinseco della linea del tempo: 1'unilateralità dello sguardo di chi osserva, senza per questo metterne in dubbio l'utilità. Non sarà difficile aprire la discussione sul modo di rappresentare questi percorsi storici "altri" rispetto a quelli più conosciuti e trovar loro una collocazione in parallelo al percorso mediterraneo. E sarà gioco-forza indispensabile allargare la riflessione ai luoghi e ai popoli di cui non si conoscono civiltà periodizzanti, o perché ancora da scoprire, o perché non si affermarono sugli altri, o ancora perché vissero vite comuni nelle loro epoche, come la maggior parte dei popoli che la storia non riesce a mostrare.

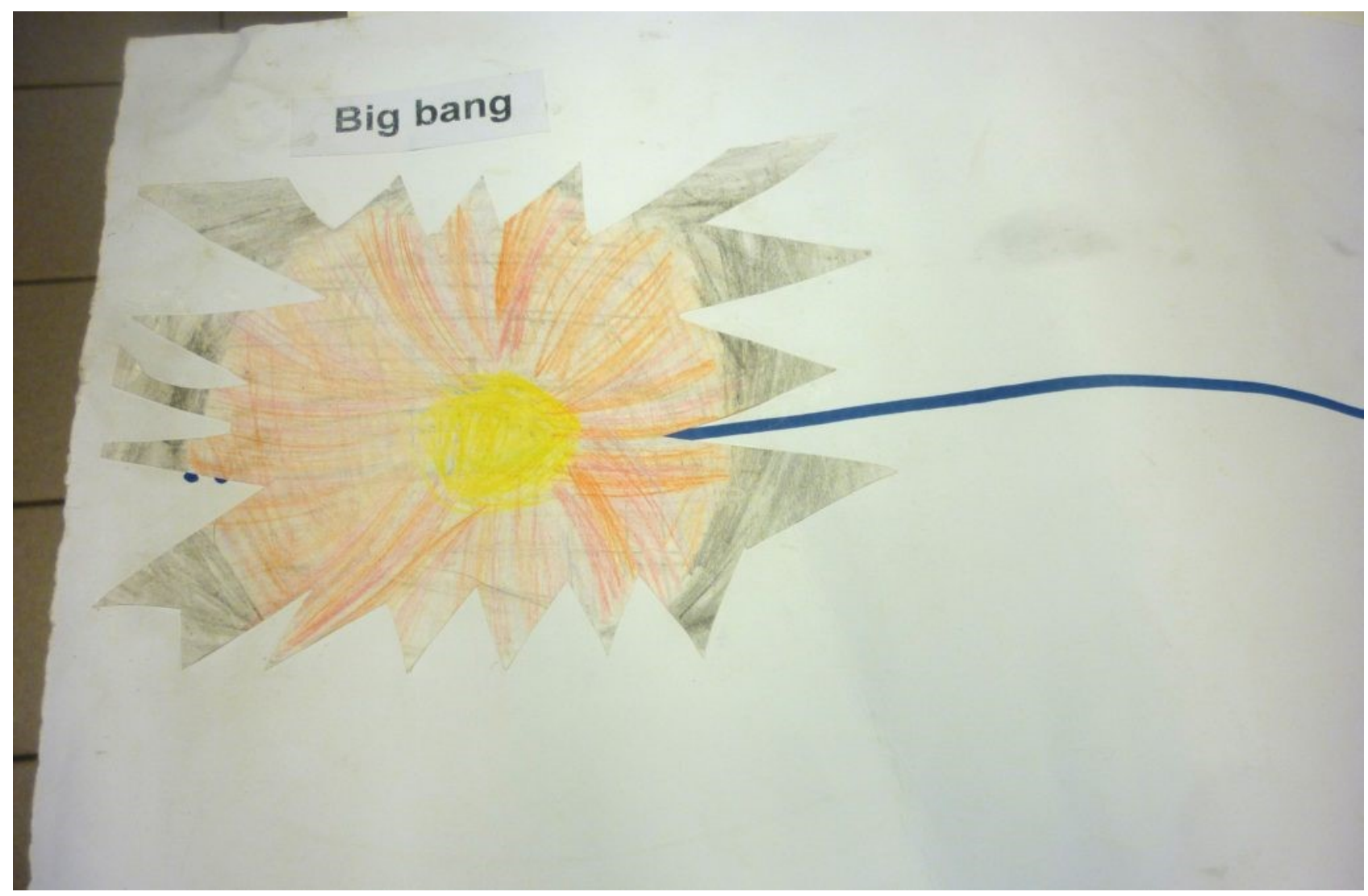

Infine, sempre nelle classi alte, sarebbe utile preparare accanto alla linea del tempo generale che va dal Big bang a oggi una seconda linea che ospiti gli avvenimenti degli ultimi 120 anni. La contemporaneità è una prospettiva importante da visualizzare nei suoi dettagli: una linea ad essa dedicata dovrebbe avere una scala maggiore ed è meglio predisporla secondo intervalli regolari affinché sia più facile da padroneggiare. 
Anche in questo caso la discussione libera potrà essere la sorgente principale per fare scaturire gli avvenimenti da collocare sulla linea, ma in questo caso il controllo della posizione - la datazione - potrà essere affidato ai bambini attraverso ricerche sui libri o sul web. Qui l'intervento dell'insegnante dovrà integrare, qualora non emergano dalla discussione, gli eventi periodizzanti che ormai sono indispensabili per orientarsi nel Novecento (guerre mondiali, caduta del muro di Berlino, ma forse anche il 1968) e nel tempo proporre nuovi elementi (ad esempio fotografie di persone che indossano il costume da bagno servirà a indicare una trasformazione delle mentalità $\mathrm{e}$ dell'abbigliamento).

La nuova linea potrà ospitare le immagini legate agli avvenimenti che emergono quotidianamente ragionando di attualità. Parlando della Shoah sarà utile collocarla al culmine della Seconda guerra mondiale, parlando di inquinamento aereo sarà utile incollare una foto del primo volo dei fratelli Wright, parlando di consumismo del mondo occidentale sarà interessante parlare del Boom economico e ritagliare un frigorifero o una Cinquecento da incollare alla fine degli anni Cinquanta.

Anche se è evidente che compiere tali azioni non significhi ancora comprendere quel passato, è utile a sottolineare che gli aspetti del presente non sono comprensibili senza la conoscenza della loro storia.

\section{Conclusione}

«Che aspetto ha la storia? Come disegnereste il tempo?».

Rosemberg e Grafton partivano da queste domande guida per esplorare la storia delle rappresentazioni grafiche del tempo. Come abbiamo visto dall'analisi degli sforzi compiuti nel passato per la messa a punto dei modelli culturali si possono trarre stimoli interessanti per ripercorrere tali sforzi nel campo dell'apprendimento e dell'apprendistato culturale delle generazioni in formazione.

Marc Twain, grande appassionato di storia e cronologia e inventore di giochi per la memorizzazione delle date, conosceva bene l'importanza delle immagini per favorire questi processi, e con le sue figlie si spinse a cercare di rendere la storia non solo visibile ma anche «percorribile». Insegnò loro «a suddividere una strada o un sentiero in segmenti di uguale lunghezza, a chiamare "anni" quelle lunghezze, e poi a piantare dei paletti in corrispondenza dei momenti chiave» (Rosemberg e Grafton, 245).

Perché non seguirne la suggestione? Certo, non tutte le scuole italiane posseggono un giardino, ma, in attesa che i discorsi di Greta Thunberg abbiano un effetto strutturale sulla vivibilità delle nostre città, ci toccherà «periodizzare» i cementati percorsi casa-scuola.

\section{Bibliografia}

- Rosenberg D., Grafton A., Cartografie del tempo. Una storia della linea del tempo, Torino, Einaudi, 2012.

- Todeschini G., La storia umana in una mappa, «il manifesto», 27 settembre 2012

- Istituto piemontese per la storia della resistenza e dell'età contemporanea, Storia facendo. Spunti operativi per un percorso di storia a partire dalla prima classe della scuola elementare, a cura del Gruppo di lavoro sulla valutazione per la formazione, 2012, $<$ http://www.istoreto.it/storia facendo/index.htm $>$.

- Istituto piemontese per la storia della resistenza e dell'età contemporanea, Storia facendo 2. Spunti operativi per un percorso di storia a partire dalla prima classe della scuola 
elementare, a cura del Gruppo di lavoro sulla valutazione per la formazione, 2018, $<\underline{\text { http://www.istoreto.it/event/storia-facendo-2-spunti-operativi-per-un-percorso-di-storia-di- }}$ classe-seconda-della-scuola-primaria/ $>$. 